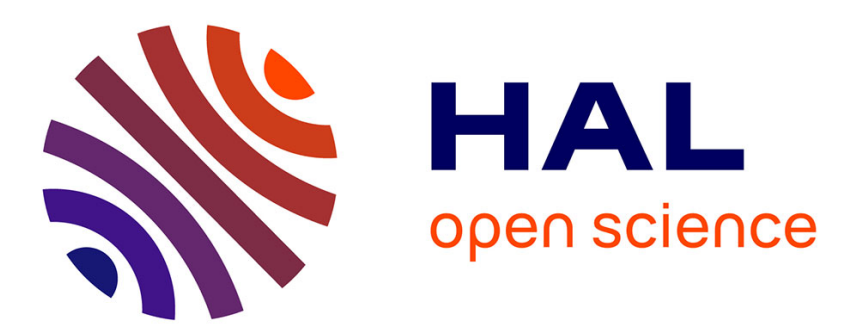

\title{
Dynamics of long-wavelength phason fluctuations in the i-Al-Pd-Mn quasicrystal
}

Sonia Francoual, Frédéric Livet, Marc de Boissieu, Flora Yakhou, Françoise Bley, Antoine Létoublon, René Caudron, Joseph Gastaldi, R. Currat

\section{To cite this version:}

Sonia Francoual, Frédéric Livet, Marc de Boissieu, Flora Yakhou, Françoise Bley, et al.. Dynamics of long-wavelength phason fluctuations in the i-Al-Pd-Mn quasicrystal. Philosophical Magazine, 2006, 86 (06-08), pp.1029-1035. 10.1080/14786430500263496 . hal-00513584

\section{HAL Id: hal-00513584 \\ https://hal.science/hal-00513584}

Submitted on 1 Sep 2010

HAL is a multi-disciplinary open access archive for the deposit and dissemination of scientific research documents, whether they are published or not. The documents may come from teaching and research institutions in France or abroad, or from public or private research centers.
L'archive ouverte pluridisciplinaire HAL, est destinée au dépôt et à la diffusion de documents scientifiques de niveau recherche, publiés ou non, émanant des établissements d'enseignement et de recherche français ou étrangers, des laboratoires publics ou privés. 


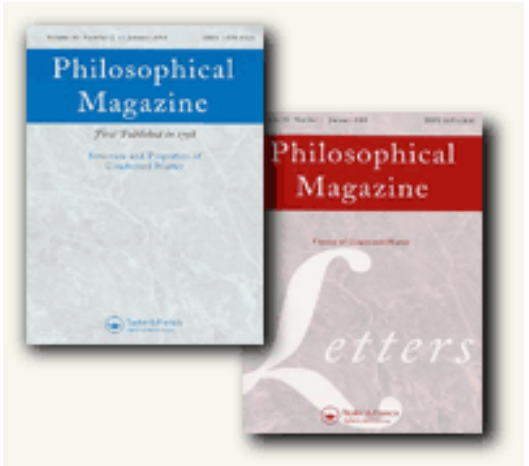

\section{Dynamics of long-wavelength phason fluctuations in the i- Al-Pd-Mn quasicrystal}

\begin{tabular}{|c|c|}
\hline Journal: & Philosophical Magazine \& Philosophical Magazine Letters \\
\hline Manuscript ID: & TPHM-05-May-0269.R1 \\
\hline Journal Selection: & Philosophical Magazine \\
\hline $\begin{array}{r}\text { Date Submitted by the } \\
\text { Author: }\end{array}$ & 15-Jul-2005 \\
\hline Complete List of Authors: & $\begin{array}{l}\text { Francoual, Sonia; LTPCM, ENSEEG / INPG, UMR CNRS 5614; ILL } \\
\text { Livet, Frédéric; LTPCM } \\
\text { de Boissieu, Marc; LTPCM } \\
\text { Yakhou, Flora; ESRF } \\
\text { Bley, Françoise; LTPCM } \\
\text { Létoublon, Antoine; CEA-Grenoble, DRFMC/SP2M } \\
\text { Caudron, René; ONERA, LEM } \\
\text { Gastaldi, Joseph; CRMC2-CNRS } \\
\text { Currat, R.; ILL }\end{array}$ \\
\hline Keywords: & X-ray, quasicrystals \\
\hline Keywords (user supplied): & phasons, coherent X-ray \\
\hline
\end{tabular}

\section{(s) ScholaroNE" \\ Manuscript Central}


Dynamics of long-wavelength phason fluctuations in the i-Al-Pd-Mn quasicrystal

\author{
S. FRANCOUAL ${ }^{*} \dagger \neq$, F. LIVET $\dagger$, M. DE BOISSIEU $\dagger$, F. YAKHOU§, F. BLEY $\dagger$, \\ A. LÉTOUBLONII, R. CAUDRONII\&, J. GASTALDI\#, R. CURRAT $\$$ \\ †LTPCM, ENSEEG, INPG, UMR CNRS 5614, 38402 Saint Martin d'Hères, FRANCE \\ \$ILL, 6, rue Jules Horowitz, BP 156, 38042 Grenoble Cedex 9, FRANCE \\ §ESRF, 6 rue Jules Horowitz, BP 220, 38043 Grenoble Cedex 9, FRANCE \\ ICEA-Grenoble, DRFMC/SP2M, 17 rue des Martyrs, 38054 Grenoble Cedex 9, FRANCE \\ IONERA - LEM, 29 av. de la Division Leclerc, F-92322 Chatillon Cedex, FRANCE \\ \&LLB, CEA-CNRS UMR12, CE Saclay, 91191 Gif-sur-Yvette Cedex, FRANCE \\ \#CRMC2-CNRS, Campus de Luminy, case 913, 13288 Marseille Cedex 09, FRANCE
}

\begin{abstract}
We report on the dynamics of phason modes in the i-Al-Pd-Mn icosahedral quasicrystal, measured between room temperature and $650^{\circ} \mathrm{C}$, using the $\mathrm{X}$ ray Intensity Fluctuation Spectroscopy technique (XIFS). Up to $500^{\circ} \mathrm{C}$, the auto-correlation function $\mathfrak{I}(\boldsymbol{q}, t)$ displays almost no time evolution as expected for frozen-in phason fluctuations at low temperature. At higher temperatures, $\Im(\boldsymbol{q}, t)$ follows a single exponential time decay from which the characteristic time $\tau_{c}(\boldsymbol{q})$ is extracted. These results are compared with the expected shape of $\mathfrak{I}(\boldsymbol{q}, t)$ as derived from the expressions of the eigenvalues and eigenvectors of the $\mathbf{C}_{\perp \perp}(\boldsymbol{q})$ phason dynamical matrix. In agreement with the hydrodynamic theory of quasicrystals which predicts phasons with diffusive character, we find that $\tau_{\mathrm{c}}(\boldsymbol{q})$ varies linearly with $q^{-2}$ at $650^{\circ} \mathrm{C}$. The corresponding diffusion coefficient is $2.2( \pm 0.5) \times 10^{-18} \mathrm{~m}^{2} \cdot \mathrm{s}^{-1}$ and the activation energy is estimated around $2.3( \pm 1) \mathrm{eV}$.
\end{abstract}

Keywords: Quasicrystals; Phasons; Coherent X-ray;

\begin{abstract}
\$1. Introduction
Quasicrystals are long-range ordered structures having rotational symmetries incompatible with translational invariance (see [1] and refs. therein). In the superspace description of icosahedral phases, the periodicity is restored in a 6-dimensional (6D) space, $\mathbf{E}$. There, the lattice is a $6 \mathrm{D}$ cube decorated with $3 \mathrm{D}$ atomic domains lying in $\mathbf{E}_{\perp}$, the perpendicular $3 \mathrm{D}$ space. $\mathbf{E}_{\perp}$ is the phason space, oriented in such a way as to be orthogonal to the physical space $\mathbf{E}_{/ /}$and incommensurate with $\mathbf{E}$. A section of $\mathbf{E}$ by $\mathbf{E}_{/ /}$generates the 3D atomic structure. Hydrodynamics is concerned with those excitations whose frequency $\omega \rightarrow 0$ as the wavevector $q \rightarrow 0$. The symmetries leaving the system's free energy invariant are associated with $\boldsymbol{u}$ and $\boldsymbol{w}$, the two uniform displacement fields along $\mathbf{E}_{/ /}$and $\mathbf{E}_{\perp}$, respectively [2]. The degeneracy with respect to the variable $\boldsymbol{w}$ introduces 3 Goldstone phason modes in addition to the 3 acoustic phonons associated with $\boldsymbol{u}$. Long-wavelength phasons are diffusive modes associated with discrete atomic rearrangements in physical space. In a simplified picture, a phason can be seen as a damped sine-wave fluctuation with wavevector $\boldsymbol{q}$ along $\mathbf{E}_{/ /}$and polarisation vector $\boldsymbol{e}_{\perp}(\boldsymbol{q})$ along $\mathbf{E}_{\perp}$. Neglecting phononphason interactions, 3 phason eigenmodes $(k=1,2,3)$ can be defined for a given vector
\end{abstract}

*Corresponding author : sonia.francoual@ltpcm.inpg.fr 
$\boldsymbol{q}$, each having its own relaxation time $\tau_{\perp, k}(\boldsymbol{q})$ and coefficient of diffusion $D_{\perp, k}(\widetilde{\boldsymbol{q}})$, given by :

$$
\left(\tau_{\perp, k}(\boldsymbol{q})\right)^{-1}=\Gamma_{\boldsymbol{w}} \times K_{\perp, k}(\boldsymbol{q})=D_{\perp, k}(\tilde{\boldsymbol{q}}) \times q^{2}
$$

where the subscript $\perp$ stands for the phason related variables. $\Gamma_{\mathrm{w}}$ is a kinetic coefficient and $\tilde{\boldsymbol{q}}=\boldsymbol{q} \wedge q \mid K_{\perp, k}(\boldsymbol{q})$ and $\boldsymbol{e}_{\perp, k}(\boldsymbol{q})$ are, respectively, the eigenvalues and eigenvectors of the $3 \times 3 \mathbf{C}_{\perp \perp}$ phason dynamical matrix. The $\mathbf{C}_{\perp \perp}$ matrix elements depend on $K_{l}$ and $K_{2}$, the phason generalised elastic constants, and on the components of $\boldsymbol{q}$.

Long-wavelength phasons lead to additional diffuse scattering near Bragg peaks in reciprocal space [3-5]. In the framework of elasticity theory, generalised to icosahedral phases, the phason diffuse intensity, noted $I_{\perp}$, at a distance $\boldsymbol{q}$ from a Bragg peak with component $\boldsymbol{Q}_{/ /}$in reciprocal space writes as :

$$
I_{\perp}\left(\boldsymbol{Q}_{/ /}+\boldsymbol{q}\right)=\sum_{k} I_{\perp, k}\left(\boldsymbol{Q}_{/ /}+\boldsymbol{q}\right)=I_{\text {Bragg }}\left(\boldsymbol{Q}_{/ /}\right) \times k_{B} \times T \times \sum_{k}\left(\boldsymbol{e}_{\perp, k}(\boldsymbol{q}) \cdot \boldsymbol{Q}_{\perp}\right)^{2} / K_{\perp, k}(\boldsymbol{q})
$$

where $\boldsymbol{Q}_{\perp}$ is the conjugate component of $\boldsymbol{Q}_{/ /}$in perpendicular space, $k_{B}$ the Boltzmann constant and $T$ the temperature. Neutron and $\mathrm{X}$-ray scattering experiments performed on iAl-Pd-Mn have evidenced such a phason diffuse intensity response [6-7]. In particular, insitu studies show a decrease of the diffuse intensity, $I_{\perp}$, simultaneously with an increase of the Bragg peak intensity, $I_{\text {Bragg, }}$, as the temperature is increased from $600^{\circ} \mathrm{C}$ to $770^{\circ} \mathrm{C}$. This reversible temperature evolution is found related to a softening of the phason elastic constant $K_{l}$, the ratio $K_{2} / K_{l}$ changing from a value -0.53 below $600^{\circ} \mathrm{C}$ to a value -0.40 at $770^{\circ} \mathrm{C}$.

In the present paper, we report on the dynamics of phasons having a wavevector $\mathbf{q}$ along the $(-\tau, 1,0)$ direction, where $\tau$ is the golden mean defined as $\tau=(1+\sqrt{5}) / 2$. The diffuse intensity is measured using a coherent X-ray beam so that it exhibits sharp intensity fluctuations called speckles. Speckles are sensitive to the time evolution of the atomic density so that any time dependence induced by phason fluctuations will result in a correlated time dependence for the speckle intensity distribution. The intensity-intensity correlation function $\mathfrak{I}(\boldsymbol{q}, t)$ is defined as:

$$
\mathfrak{J}(\boldsymbol{q}, t)=\left\langle I\left(\boldsymbol{q}, t^{\prime}\right) \times I\left(\boldsymbol{q}, t^{\prime}+t\right)\right\rangle_{t^{\prime}} /\left(\left\langle I\left(\boldsymbol{q}, t^{\prime}\right)\right\rangle_{t^{\prime}}\right)^{2}=1+\beta \times g(\boldsymbol{q}, t)
$$

with $\beta$ the degree of coherence of the beam and $g(\boldsymbol{q}, t)$ the function which characterises the time dependence of the fluctuation. For a diffusive process, $g(\boldsymbol{q}, t)=\exp \left(-2 t / \tau_{\mathrm{c}}(\boldsymbol{q})\right)$ where $\tau_{\mathrm{c}}(\boldsymbol{q})$ is a characteristic time $\mathrm{e}^{\dagger}$.

\section{§2. Experimental}

The coherent X-ray scattering experiment was carried out on the ID20 beam line at the ESRF in Grenoble following the set-up described in $[8,9]$. The incoming energy is set to $8 \mathrm{keV}$, with an energy resolution $\Delta \lambda \lambda=1.3 \times 10^{-4}$, yielding a longitudinal coherence length $\left(\lambda^{2} / \Delta \lambda\right) \sim 1.2 \mu \mathrm{m}$. A $10 \mu \mathrm{m}$ collimating pinhole before the sample allows for a $\sim 10$ $\mu \mathrm{m}$ transverse coherence length together with a $5 \times 10^{8}$ photons/s flux at the sample position. Diffraction patterns were recorded on a Princeton Instrument Direct Illumination 2D CCD acting as a 2D X-ray photon counter [10]. About 500 frames were recorded at each temperature with a time interval of a few seconds $(<10 \mathrm{~s})$ between successive frames.

The sample is a $1 \mathrm{~mm}$ thick $\mathrm{Al}_{68.2} \mathrm{Pd}_{22.8} \mathrm{Mn}_{9}$ plate-shaped single-grain with a 5fold axis perpendicular to its surface, extracted from a Czochralski grown mother ingot

\footnotetext{
${ }^{\dagger}$ In a previous paper [8], we overlooked the factor 2 which appears in the exponent.
} 
[11]. It was annealed for 8 days at $780^{\circ} \mathrm{C}$ under ultrahigh vacuum and then slowly cooled down in order to guaranty the surface stability during the X-ray measurements [12]. After annealing, the sample surface displays flat terraces whose size, between 50 and $100 \mu \mathrm{m}$, is sufficient to focus the $10 \mu \mathrm{m}$ beam on it. The sample is placed in a furnace and heated up under secondary vacuum.

Measurements were performed near the 7/11 reflection having a Bragg angle $2 \theta$ $\sim 24^{\circ}$ and a component $\boldsymbol{Q}_{/ /}$along the $(1, \tau, 0) 5$-fold axis. The partial coherence of the beam $\beta$ is $5 \%$. From room temperature up to $650^{\circ} \mathrm{C}$, several $\boldsymbol{q}$ values were probed by rotating the $\theta$ incident angle with a $0.1^{\circ}$ step which corresponds to a step $q \sim 0.003 \AA^{-1}$ along the $(-\tau, 1,0)$ direction in reciprocal space.

\section{§3. Results}

Figure 1 shows a typical diffraction pattern obtained at a position $q=0.0125 \AA^{-1}$ away from the 7/11 Bragg peak. The simulation of the diffuse intensity distribution, shown on the right panel, is in good agreement with the measurement, insuring that the observed diffuse signal is due to phason modes. In order to improve statistics, $\Im(\boldsymbol{q}, t)$ must be averaged over a set of equivalent $\boldsymbol{q}$ vectors. Given the anisotropy of the phason diffuse intensity distribution, we considered only an average performed over a circle close to the $q / /(-\tau, 1,0)$ wavevector at the center of the CCD. '[insert figure 1 about here]'

From room temperature up to $500^{\circ} \mathrm{C}, \Im(\boldsymbol{I}, t)$ is found almost constant over $1000 \mathrm{~s}$, as shown in figures $2 \mathrm{a}$ and $2 \mathrm{~b}$. The temperature range between $500^{\circ} \mathrm{C}$ and $600^{\circ} \mathrm{C}$ is a transient regime in which some dynamics sets up. The time evolution is however very slow as can be seen in Fig. $2 \mathrm{c}$ and only few data were thus recorded. At $650^{\circ} \mathrm{C}, \Im(\boldsymbol{T}, t)$ displays a clear exponential time decay from which the characteristic time $\tau_{c}$ is extracted (see Figs. 2(d-f)). '[insert figure 2 about here]' '[insert figure 3 about here]'

The observed evolutions can be related to the dynamics of phason modes. Below $500^{\circ} \mathrm{C}$, the absence of dynamics would be the signature of frozen-in phason fluctuations as reported in [7-8]. The temperature activation occurs between $500^{\circ} \mathrm{C}$ and $600^{\circ} \mathrm{C}$. Above

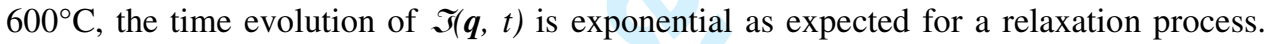
Fig. 3 is a plot of the decay rate, $\left(1 / \tau_{c}\right)$, as a function of $q^{2}$ at $650^{\circ} \mathrm{C}$ In agreement with equation (1), the dependence is linear which demonstrates that phason modes have diffusive character. The corresponding diffusion coefficient at $650^{\circ} \mathrm{C}$ is $D_{\perp}(\widetilde{\boldsymbol{q}})=2.2( \pm$ $0.5) \times 10^{-18} \mathrm{~m}^{2} \cdot \mathrm{s}^{-1 \ddagger}$. Between $600^{\circ} \mathrm{C}$ and $650^{\circ} \mathrm{C}, \tau_{\mathrm{c}}$ is divided by a factor $\sim 5$ at same $q$ value (see Figs.3c and 3e). This leads to an activation energy $E_{\mathrm{a}}=2.3( \pm 1) \mathrm{eV}$, assuming an Arrhenius-type behaviour.

\section{§4. Discussion and conclusions}

From hydrodynamic theory, for each $\boldsymbol{q}$, one expects to observe three phason modes, each with its own relaxation time. The behaviour of the function $\Im(q, t)$ should thus reflect the existence of these three relaxation times through a more complex time evolution than the single exponential time decay that is experimentally observed. By considering the intensity and relaxation time of each mode, we demonstrate, in the following, that in fact, for this particular phason wavevector direction, two modes are selected and that the resulting time evolution of $\mathfrak{I}(\boldsymbol{q}, t)$ is close to a single-exponential decay.

\footnotetext{
* The value of $D_{\perp}(\tilde{\boldsymbol{q}})$ differs numerically by a factor $8 \pi^{2}$ from the value quoted in ref [8].
} 
The diffuse intensity is measured at the position $\left(\boldsymbol{Q}_{/ /}+\boldsymbol{q}\right)$ where $\boldsymbol{q}$ is along $(-\tau, 1,0)$ and $\boldsymbol{Q}_{/ /}$ along $(1, \tau, 0)$. In that direction of $\boldsymbol{q}$, there exists a basis $\boldsymbol{e}_{\perp, k}(\boldsymbol{q})$ where $\mathrm{C}_{\perp \perp}(\boldsymbol{q})$ is diagonal with eigenvalues $K_{\perp, k}(\boldsymbol{q})$ :

$$
\boldsymbol{q} / /(-\tau, 1,0) \rightarrow\left\{\begin{array}{c}
K_{\perp, 1}(\boldsymbol{q})=\left[K_{1}+(\tau-1 / 3) \times K_{2}\right] \times q^{2}, \quad \boldsymbol{e}_{\perp, 1}(\boldsymbol{q}) / /(-1 / 2,1,0) \\
K_{\perp, 2}(\boldsymbol{q})=\left[K_{1}-(1 / \tau+1 / 3) \times K_{2}\right] \times q^{2}, \quad \boldsymbol{e}_{\perp, 2}(\boldsymbol{q}) / /(1,1 / 2,0) \\
K_{\perp, 3}(\boldsymbol{q})=\left[K_{1}-(1 / 3) \times K_{2}\right] \times q^{2}, \quad \boldsymbol{e}_{\perp, 3}(\boldsymbol{q}) / /(0,0,1)
\end{array}\right\}
$$

The phason diffuse scattering intensity is the sum of the three contributions $\mathrm{I}_{\perp, k}$ as expressed in equation (2). The scalar products $\left(\boldsymbol{Q}_{\perp} \cdot \boldsymbol{e}_{\perp, k}\right)$ act as selection rules. Since $\boldsymbol{Q}_{\perp}$ lies along $(-\tau, 1,0)$ in $\mathbf{E}_{\perp}$ space, it follows that $\left(\boldsymbol{Q}_{\perp} \cdot \boldsymbol{e}_{\perp, 3}\right)$ is zero and the mode 3 does not contribute to the intensity. We can estimate the weight of the $1^{\text {st }}$ and $2^{\text {nd }}$ modes from the value of $K_{2} / K_{1}$. We find $I_{\perp, 1} \sim I_{\perp, 2}$ at $770^{\circ} \mathrm{C}$ and $I_{\perp, 1} \sim 2 I_{\perp, 2}$ below $600^{\circ} \mathrm{C}$. We thus select two modes which contribute with comparable weights to the overall diffuse intensity at all temperatures.

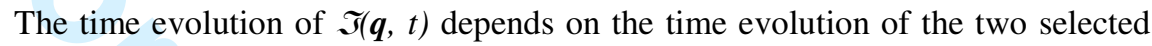
phason modes. More precisely, in definition (3), $g(\boldsymbol{q}, t)$ writes as [13] :

$$
g(\boldsymbol{q}, t)=\left[\left(I_{\perp, 1} / I_{\perp}\right) \times \exp \left(-t / \tau_{\perp, 1}(\boldsymbol{q})\right)+\left(I_{\perp, 2} / I_{\perp}\right) \times \exp \left(-t / \tau_{\perp, 2}(\boldsymbol{q})\right)\right]^{2}
$$

The phason relaxation times $\tau_{\perp, l}$ and $\tau_{\perp, 2}$ depend on $\Gamma_{w}$ and $K_{\perp, k}$ (see equation (1)). Both parameters have their own temperature behaviour. In the diffuse scattering measurements, we probe the temperature dependence of the $K_{l}$ and $K_{2}$ elastic constants only, whereas in the present experiment, we measure directly $\tau_{\perp}$ and thus get the complete temperature information. Assuming that the kinetic coefficient $\Gamma_{w}$ is identical for the three phasons, we can estimate that $\tau_{\perp, 1} \sim 5 \tau_{\perp, 2}$ at low temperature while $\tau_{\perp, 1} \sim 3 \tau_{\perp, 2}$ at $770^{\circ} \mathrm{C}$. Given the intensity and decay time ratios obtained between the $1^{\text {st }}$ and $2^{\text {nd }}$ phason modes, numerical simulations show that, within the statistical accuracy of our data, it is not possible to distinguish the time evolution given by equation (5) from a pure single exponential decay. The characteristic decay time $\tau_{c}(\boldsymbol{q})$ that is then extracted is an effective relaxation time averaged over the two selected phason modes.

At $650^{\circ} \mathrm{C}$, the phason diffusion coefficient $D_{\perp}(\tilde{\boldsymbol{q}})$ is found equal to $2.2 \times 10^{-18}$ $\mathrm{m}^{2} . \mathrm{s}^{-1}$. When compared with the atomic self-diffusion of the Pd and Mn elements obtained at same temperature in i-Al-Pd-Mn by the radiotracer techniques [14], we find 4 orders of magnitude of difference between the two types of relaxation processes, as $D_{P d} \sim 4 \times D_{M n} \sim$ $6 \times 10^{-14} \mathrm{~m}^{2} \cdot \mathrm{s}^{-1}$. On the other hand, our result compares well with mechanical spectroscopy measurements [15] in which a slow relaxation process involving the collective motion of a large number of atoms was identified. Phasons are believed to play a key role in the high temperature mechanical properties of quasicrystals. In particular, it has been observed that the motion of dislocations in i-Al-Pd-Mn [16] is followed by the formation of planar faults associated with the phason-displacement field $\mathbf{w}$ around the dislocation line. A possible scenario is that these phason walls disappear as phason modes are activated at high temperature. Such annealing has been recently reported in [17]. The complete dispersion of a $\sim 0.1 \mu \mathrm{m}$ thick phason wall is achieved after $12 \mathrm{~s}$ at $700^{\circ} \mathrm{C}$. Assuming that the above time scale is of the order of $\tau_{\perp}$, from the values of $E_{\mathrm{a}}$ and $D_{\perp}$, we obtain a characteristic phason length scale $\lambda_{\perp}(=2 \pi / q)$ of $685 \AA$ at $700^{\circ} \mathrm{C}$. This is of the same order of magnitude as the phason wall thickness which indicates that both results are compatible.

To summarise, we measured the dynamics of long-wavelength phasons in the iAlPdMn quasicrystal using coherent X-rays. The diffusive character of phason modes, predicted by hydrodynamic theory, is experimentally evidenced. The diffusion coefficient 
is found to be $2.2( \pm 0.5) \times 10^{-18} \mathrm{~m}^{2} . \mathrm{s}^{-1}$ at $650^{\circ} \mathrm{C}$ with an activation energy equal to $2.3( \pm 1)$ $\mathrm{eV}$.

\section{References}

[1] A. Yamamoto, Acta.Cryst. A52 (1996)

[2] T. C. Lubensky, Introduction to Quasicrystals (ed. by M. V. Jaric, 1988), pp. 199-277

[3] M. V. Jaric and D. R. Nelson, Phys. Rev. B 379 (1988)

[4] M. Widom, Phil. Mag. Lett. 645 (1991)

[5] Y. Ishii, Phys. Rev. B. 4510 (1992)

[6] M. de Boissieu, M. Boudard, B. Hennion, et al., Phys. Rev. Lett. 751 (1995)

[7] M. Boudard, M. de Boissieu, A. Létoublon, et al., Europhys. Lett. 333 (1996)

[8] S. Francoual, F. Livet, M. de Boissieu, et al., Phys. Rev. Lett. 9122 (2003)

[9] A. Létoublon, M. de Boissieu, M. Boudard, et al., Phil. Mag. Lett. 814 (2001)

[10] F. Livet, F. Bley, A. Létoublon, et al., J. Synchrotron Rad. 5 (1998)

[11] M. Boudard, E. Bourgeat-Lami, M. de Boissieu, et al., Phil. Mag. Lett. 7111 (1995)

[12] F. Schmithüsen, PhD thesis, University Joseph Fourier, Grenoble, France (2001)

[13] B. Berne and R. Pecora, Dynamic Light Scattering, ed. John Wiley \& Sons (1976)

[14] H. Mehrer, R. Galler, W. Frank, et al., Diffusion in Quasicrystals, Quasicrystals Structure and Physical Properties, ed. by H.-R. Trebin, WILEY-VCH, Weinheim (2003)

[15] B. Damson, M. Weller, M. Feuerbacher, et al., Mater. Sci. Eng. 294-296 (2000)

[16] D. Caillard, G. Vanderschaeve, L. Bresson, et al., Phil. Mag. A 801 (2000)

[17] F. Mompiou, D. Caillard and M. Feuerbacher, Phil. Mag. 84 25-26 (2004) 
Figure 1: Left: Diffraction pattern obtained at $650^{\circ} \mathrm{C}$ at the distance $q=0.0125 \AA^{-1}$ from the 7/11 Bragg peak. 400 frames were measured with a $7 \mathrm{~s}$ time interval. The diffuse intensity distribution is anisotropic in agreement with an intensity response due to phasons. Right: Simulation of the phason diffuse scattering in the i-Al-Pd-Mn phase in the relevant part of reciprocal space following the model of Jaric [5] with $K_{2} / K_{l}=-0.53$.

Figure 2: Time dependence of the auto-correlation function $\mathfrak{I}(\boldsymbol{q}, t)$ at different temperatures and different values of the phason wavevector $\boldsymbol{q}$. The solid line is an exponential fit to the data. $\Im(q, t)$ is constant up to $500^{\circ} \mathrm{C}(\mathrm{a}-\mathrm{b})$. At $600^{\circ} \mathrm{C}$ and $650^{\circ} \mathrm{C}, \mathfrak{J}(\boldsymbol{q}$, t) decays exponentially (c-f). At $650^{\circ} \mathrm{C}$, the decay time $\tau_{c}(\boldsymbol{q})$ decreases with $q(\mathrm{~d}-\mathrm{f})$.

Figure 3: Evolution of the inverse of the relaxation time $\left(1 / \tau_{\mathrm{c}}\right)$ as a function of $q^{2}$ with $\boldsymbol{q}$ along $(-\tau, 1,0)$, at $650^{\circ} \mathrm{C}$. The linear trend is a signature of a diffusive process allowing the determination of the phason diffusion coefficient $D_{\perp}(\tilde{\boldsymbol{q}})=2.2( \pm 0.5) \times 10^{-18} \mathrm{~m}^{2} \cdot \mathrm{s}^{-1}$. 
Figure 1

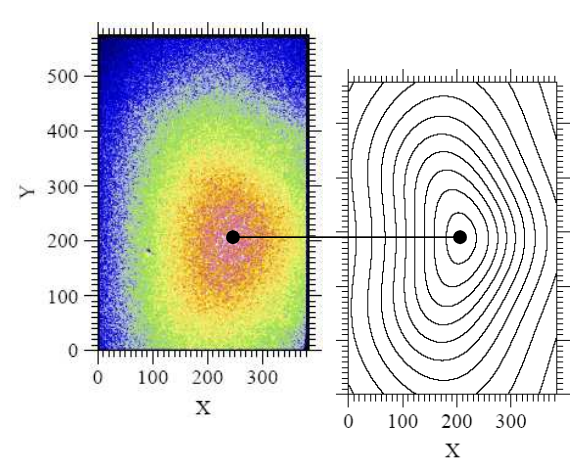

14

15

16

17

18

19

20

21

22

23

24

25

26

27

28

29

30

31

32

33

34

35

36

37

38

39

40

41

42

43

44

45

46

47

48

49

50

51

52

53

54

55

56

57

58

59

60

http://mc.manuscriptcentral.com/pm-pml 
Figure 2
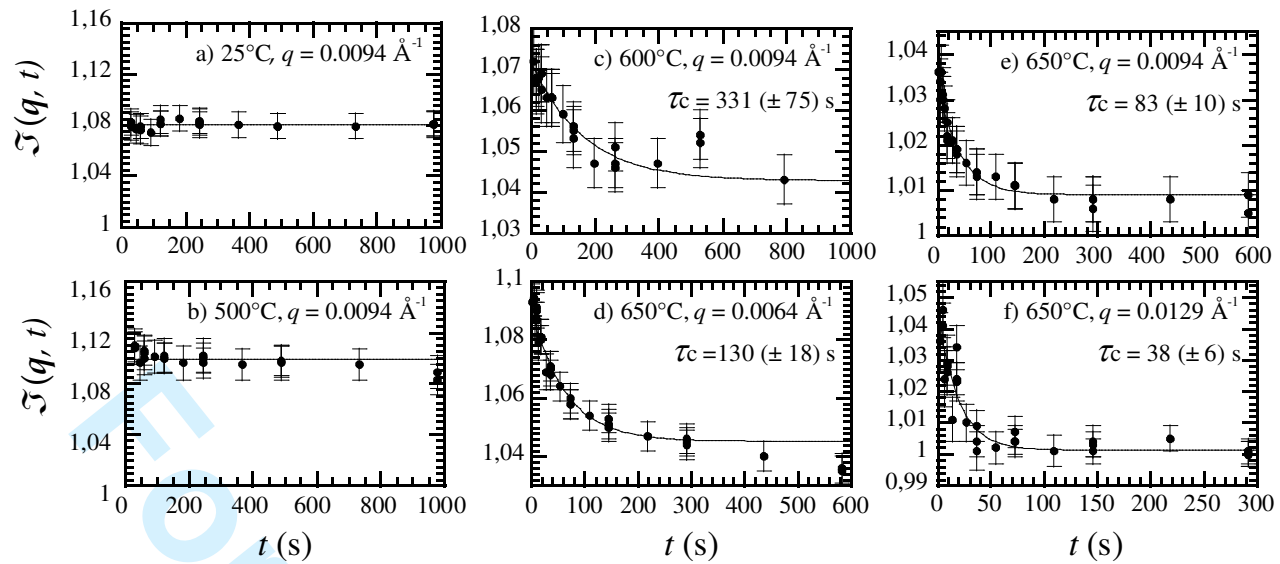

18

19

20

21

22

23

24

25

26

27

28

29

30

31

32

33

34

35

36

37

38

39

40

41

42

43

44

45

46

47

48

49

50

51

52

53

54

55

56

57

58

59

60 
Figure 3

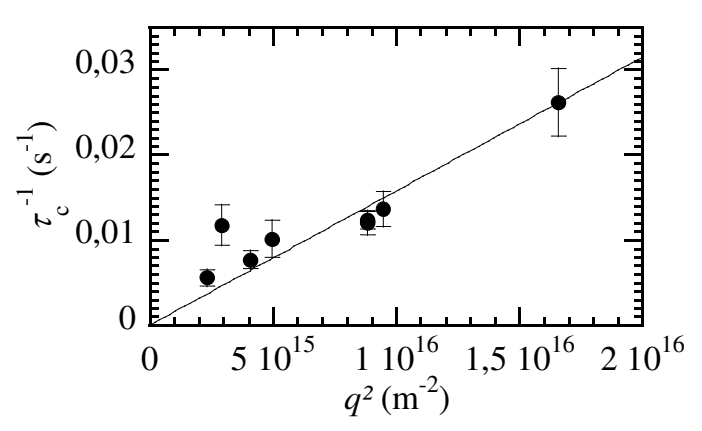

13

14

15

16

17

18

19

20

21

22

23

24

25

26

27

28

29

30

31

32

33

34

35

36

37

38

39

40

41

42

43

44

45

46

47

48

49

50

51

52

53

54

55

56

57

58

59

60

http://mc.manuscriptcentral.com/pm-pml 